\title{
Postmortem Methamphetamine Distribution
}

lain M. Mclntyre*, Catherine Hamm and Ernie Bader

Director and Chief Toxicologist, San Diego County Medical Examiner's Office, 5570 Overland Ave., CA, USA

\begin{abstract}
Methamphetamine is a commonly abused central nervous system stimulant. The San Diego County Medical Examiner's Office has seen increasing positive methamphetamine cases that do not have a suitable blood sample for analysis. We compare liver, vitreous, and central blood levels of methamphetamine and the metabolite amphetamine to levels in peripheral blood samples in 18 medical examiner cases. Specimens were screened by ELISA and confirmed by GCMS analysis following liquid-liquid extraction. Methamphetamine central blood to peripheral blood ratios averaged $1.61( \pm 0.48)$, vitreous to peripheral blood $1.63( \pm 0.75)$ and liver to peripheral blood $5.68( \pm 2.32)$. The data suggests that in cases where blood is not available, vitreous and liver specimens are useful in confirming methamphetamine use.
\end{abstract}

Keywords: Postmortem; Methamphetamine; Amphetamine; Vitreous; Liver; Blood

\section{Introduction}

Methamphetamine is a highly addictive central nervous system stimulant that can be injected, snorted, smoked, or ingested orally. Although methamphetamine is available in $5 \mathrm{mg}$ tablets by prescription for the treatment of attention deficit disorder [1], it's major use is illicit. Methamphetamine is generally synthesized in clandestine laboratories. It is metabolized by $\mathrm{N}$-demethylation to amphetamine, which is also an active drug [2].

The San Diego County Medical Examiner's Office has experienced an increased number of methamphetamine cases every year in which blood samples are not available for analysis. Due to the high volume of methamphetamine positive deaths in our county we were able to study the postmortem redistribution in peripheral blood, central blood, liver and vitreous samples. In 1999, Barnhart et al. [3] studied postmortem redistribution of central and peripheral blood. Their conclusion was that peripheral blood would accurately reflect methamphetamine concentrations over elevated central blood samples. However there has not been a thorough study on levels of methamphetamine distribution for vitreous and liver samples.

This study will examine 18 postmortem cases in which peripheral blood, central blood, liver and vitreous samples were available to sample from known methamphetamine positive cases. This study presents a more comprehensive investigation of postmortem redistribution, and provides better insight on how to correlate vitreous and liver samples with blood matrices.

\section{Experimental}

\section{Sample collection and storage}

Methamphetamine positive cases with sufficient appropriate samples were chosen for this study. Peripheral blood samples were collected distally from the femoral vein by clamping off the iliac vein. Central blood was collected after the heart was removed and then the blood was drawn from that cavity. Bloods were stored in gray-top Vacutainer tubes. The upper right lobe of the liver was sampled for all liver cases. Vitreous humor samples were withdrawn from the eye with a syringe and stored in red-top Vacutainer tubes. All specimens were stored at $4^{\circ} \mathrm{C}$ until they were analyzed. Samples were extracted and analyzed 12-72 (mean: 38 ) days after autopsy.

\section{Screening}

Screening for amphetamines was accomplished by ELISA (Immunalysis kit \#211) for methamphetamine. The reference was set at $50 \mathrm{ng} / \mathrm{mL}$ of methamphetamine. Positive cases were then confirmed using the Gas Chromatography/Mass Spectroscopy (GC/MS).

\section{Materials}

Solvents (1-Chlorobutane, Methanol and Acetone) were EM Science OmniSolv ${ }^{\circledR}$ grade from VWR Inc. Pentafluoropropionic Anhydride (PFPA) was obtained from Sigma-Aldrich. Reagent grade ammonium hydroxide and hydrochloric acid were obtained from VWR Inc.

Methamphetamine, amphetamine, methamphetamine-D5 and amphetamine-D5 were obtained from Cerilliant (Austin, TX).

An aqueous working standard containing $1.0 \mathrm{mg} / \mathrm{L}$ each of methamphetamine and amphetamine was prepared. An aqueous working internal standard containing $1.0 \mathrm{mg} / \mathrm{L}$ each of methamphetamine-D5 and amphetamine-D5 was prepared.

A linear calibration curve from 0.02 to $2.0 \mathrm{mg} / \mathrm{L}$ was produced using five calibrators at $0.02,0.05,0.25,1.0$, and $2.0 \mathrm{mg} / \mathrm{L}$ made by diluting the working standard. All calibrators were prepared in deionized water.

A commercial whole blood toxicology control containing 0.10 $\mathrm{mg} / \mathrm{L}$ of methamphetamine and amphetamine obtained from UTAK Laboratories (Product \#98818), and an in-house whole blood control containing $0.10 \mathrm{mg} / \mathrm{L}$ methamphetamine and amphetamine was run with each batch of calibrators.

\section{Extraction}

Methamphetamine and amphetamine were extracted from biologicals using a modification of the procedure described by Foerster

*Corresponding author: Iain M. McIntyre, San Diego Medical Examiner's Office, 5570 Overland Ave., Suite 101 San Diego, CA. 92123, USA, Tel: 8586942907 Fax: 858495 5383; E-mail: iain.mcintyre@sdcounty.ca.gov

Received March 19, 2011; Accepted April 06, 2011; Published April 11, 2011

Citation: McIntyre IM, Hamm C, Bader E (2011) Postmortem Methamphetamine Distribution. J Forensic Res 2:122. doi:10.4172/2157-7145.1000122

Copyright: (c) 2011 Mclntyre IM. This is an open-access article distributed unde the terms of the Creative Commons Attribution License, which permits unrestricted use, distribution, and reproduction in any medium, provided the original author and source are credited. 
et al. [4]. Liver homogenates were prepared by making a 1:1 dilution with deionized water and homogenized using either a commercial blender or dispersion mixer. The sample size extracted for all standards, controls and biologicals were brought to $2.0 \mathrm{~mL}$ volume with deionized water. Working internal standard $(0.5 \mathrm{~mL})$ was added to all tubes.

Concentrated Ammonium hydroxide $(0.8 \mathrm{~mL})$ was added to each tube and mixed by vortexing. Extraction solvent (1-chlorobutane, $7 \mathrm{~mL}$ ) was added to each tube, they were capped and extracted 30 minutes on a Labquake table shaker. Tubes were centrifuged at 2800 $\mathrm{rpm}$ for 10 minutes. Organic top layer was transferred to a $16 \times 125$ screw cap tube. Back extraction was accomplished by adding $5 \mathrm{~mL}$ $1 \mathrm{~N} \mathrm{HCl}$ and extracting on a rotating wheel for 30 minutes. The tubes were then centrifuged at $2800 \mathrm{rpm}$ for 5 minutes and the organic phase was removed and discarded. To each tube, $1.5 \mathrm{~mL}$ Ammonium Hydroxide was added and vortexed for 15 seconds. Extraction solvent (1-Chlorobutane, $3.5 \mathrm{~mL}$ ) was then added to each tube and extracted 30 minutes on a Labquake tabletop shaker. After centrifuging at 2800 rpm for 5 minutes, the organic phase was transferred to clean $16 \times 125$ screw cap tubes. Extracts were derivatized with $100 \mu \mathrm{L}$ PFPA at $60^{\circ} \mathrm{C}$ for 25 minutes in a heating block. After cooling to room temperature, the extracts were evaporated just to dryness with nitrogen in a $40^{\circ} \mathrm{C}$ water bath. Extracts were reconstituted with $175 \mu \mathrm{L}$ acetone, mixed by vortexing, and then transferred to autosampler vials.

\section{Instrumentation}

One microliter splitless injections were made onto an Agilent Technologies 6890 Gas Chromatograph. The GC column was an HP-1 capillary column (Agilent Technologies $15 \mathrm{~m}, 0.25 \mathrm{~mm}$ diameter, $0.25 \mu \mathrm{m}$ film thickness) with helium as the carrier gas. The GC oven was held for 2 minutes at an initial temperature of $175^{\circ} \mathrm{C}$, ramped $25^{\circ} \mathrm{C} /$ min until it reached $150^{\circ} \mathrm{C}$ and held for 2 minutes, then increased $30^{\circ} \mathrm{C} /$ min to a final temperature of $270^{\circ} \mathrm{C}$. An Agilent $5973 \mathrm{MSD}$ was used for the selective ion monitoring (SIM). The GCMS was controlled by Chemstation software. The total chromatography time per injection was 12 minutes.

The following ions were monitored and used for measuring the internal standard: $m / z \quad 208$ for methamphetamine-D5 and $m / z \quad 194$ for amphetamine-D5. The ions monitored for quantitation: m/z 204 for methamphetamine and m/z 190 for amphetamine. The ions monitored as qualifiers: $\mathrm{m} / \mathrm{z} 118,160$ for methamphetamine and $\mathrm{m} / \mathrm{z} 91,118$ for amphetamine. Other compounds routinely detected with this method include ephedrine, pseudoephedrine, methylenedioxymethamphetamine (MDMA), methylenedioxyamphetamine (MDA), phentermine, and phenylephrine.

\section{Results and Discussion}

A total of 18 cases were sequentially collected where central blood, peripheral blood, liver and vitreous were all available. Methamphetamine concentrations for all tissues are shown in Table 1. Table 2 gives concentration ratios of methamphetamine to peripheral blood for central blood, vitreous, and liver specimens. The central blood $(\mathrm{CB})$ to peripheral blood $(\mathrm{PB})$ concentration ratio averaged 1.61 for methamphetamine (median 1.62). Liver to peripheral blood ratios averaged 5.68 (median 6.25). The data suggests that methamphetamine also distributes well into vitreous humor with an average vitreous to peripheral blood ratio of 1.63 (median 1.41).

Table 3 shows the amphetamine concentrations for all tissues. Amphetamine concentration ratios to peripheral blood for central

\begin{tabular}{|c|c|c|c|c|}
\hline Case \# & PB & CB & Vitreous & Liver \\
\hline 1 & 0.61 & 1.40 & 0.84 & 4.10 \\
\hline 2 & 0.24 & 0.48 & 0.21 & 1.60 \\
\hline 3 & 0.25 & 0.48 & 0.64 & 2.10 \\
\hline 4 & 0.38 & 0.42 & 0.53 & 0.94 \\
\hline 5 & 0.26 & 0.25 & 0.29 & 1.00 \\
\hline 6 & 0.40 & 0.78 & 0.57 & 1.80 \\
\hline 7 & 0.26 & 0.29 & 0.42 & 2.00 \\
\hline 8 & 0.33 & 0.53 & 0.89 & 3.00 \\
\hline 9 & 0.35 & 0.75 & 1.10 & 3.20 \\
\hline 10 & 0.31 & 0.73 & 0.85 & 2.20 \\
\hline 11 & 1.60 & 2.40 & 1.10 & 3.10 \\
\hline 12 & 0.42 & 0.91 & 0.45 & 1.80 \\
\hline 13 & 1.10 & 1.80 & 2.10 & 6.10 \\
\hline 14 & 0.25 & 0.30 & 0.52 & 1.50 \\
\hline 15 & 1.60 & 2.00 & 2.00 & 4.70 \\
\hline 16 & 0.60 & 1.00 & 1.00 & 3.90 \\
\hline 17 & 1.70 & 1.60 & 1.90 & 12.00 \\
\hline 18 & 0.69 & 0.78 & 0.40 & 1.60 \\
\hline
\end{tabular}

$\mathrm{PB}=$ Peripheral Blood $\mathrm{CB}=$ Central Blood

Table 1: Postmortem Distribution of Methamphetamine $(\mathrm{mg} / \mathrm{L})$ or $(\mathrm{mg} / \mathrm{kg})$.

\begin{tabular}{|c|c|c|c|}
\hline Case \# & $\mathrm{CB} / \mathrm{PB}$ & Vitreous/PB & Liver/PB \\
\hline 1 & 2.30 & 1.38 & 6.72 \\
\hline 2 & 2.00 & 0.88 & 6.67 \\
\hline 3 & 1.92 & 2.56 & 8.40 \\
\hline 4 & 1.11 & 1.39 & 2.47 \\
\hline 5 & 0.96 & 1.12 & 3.85 \\
\hline 6 & 1.95 & 1.43 & 4.50 \\
\hline 7 & 1.12 & 1.62 & 7.69 \\
\hline 8 & 1.61 & 2.70 & 9.09 \\
\hline 9 & 2.14 & 3.14 & 9.14 \\
\hline 10 & 2.35 & 2.74 & 7.10 \\
\hline 11 & 1.50 & 0.69 & 1.94 \\
\hline 12 & 2.17 & 1.07 & 4.29 \\
\hline 13 & 1.64 & 1.91 & 5.55 \\
\hline 14 & 1.20 & 2.08 & 6.00 \\
\hline 15 & 1.25 & 1.25 & 2.94 \\
\hline 16 & 1.67 & 1.67 & 6.50 \\
\hline 17 & 0.94 & 1.12 & 7.06 \\
\hline 18 & 1.13 & 0.58 & 2.32 \\
\hline mean & 1.61 & 1.63 & 5.68 \\
\hline SD & 0.48 & 0.75 & 2.32 \\
\hline median & 1.62 & 1.41 & 6.25 \\
\hline
\end{tabular}

$\mathrm{PB}=$ Peripheral Blood

$\mathrm{CB}=$ Central Blood

Table 2: Methamphetamine Distribution Ratios

blood, vitreous, and liver specimens are shown in Table 4. The CB/PB ratio averaged 1.58 (median 1.53). The liver/PB ratio mean was 8.09 (median 7.0). The vitreous to peripheral blood ratio averaged 1.82 (median 1.54).

Only five of the eighteen cases had more than twice the methamphetamine concentration in the central blood when compared to peripheral blood. Of those five, the highest difference was 2.35 times that of peripheral blood. We did not see the high ratios (up to 5) reported by Barnhart et al. [3]. The results for amphetamine distribution ratios were similar to those of methamphetamine.

The vitreous levels for both methamphetamine and amphetamine 
Citation: McIntyre IM, Hamm C, Bader E (2011) Postmortem Methamphetamine Distribution. J Forensic Res 2:122. doi:10.4172/2157-7145.1000122

Page 3 of 3

\begin{tabular}{|c|c|c|c|c|}
\hline Case \# & PB & CB & Vitreous & Liver \\
\hline 1 & 0.05 & 0.10 & 0.07 & 0.39 \\
\hline 2 & ND & ND & ND & ND \\
\hline 3 & 0.04 & 0.05 & 0.09 & $*$ \\
\hline 4 & 0.05 & 0.06 & 0.06 & 0.17 \\
\hline 5 & 0.02 & 0.02 & 0.04 & 0.14 \\
\hline 6 & 0.02 & 0.03 & 0.04 & 0.14 \\
\hline 7 & 0.04 & 0.02 & 0.09 & 0.68 \\
\hline 8 & 0.03 & 0.06 & 0.11 & 0.44 \\
\hline 9 & 0.04 & 0.10 & 0.13 & 0.47 \\
\hline 10 & 0.02 & 0.04 & 0.05 & 0.17 \\
\hline 11 & 0.18 & 0.28 & 0.12 & 0.50 \\
\hline 12 & 0.04 & 0.10 & 0.04 & 0.21 \\
\hline 13 & 0.06 & 0.11 & 0.09 & 0.40 \\
\hline 14 & 0.07 & 0.10 & 0.11 & 0.49 \\
\hline 15 & 0.16 & 0.23 & 0.17 & 0.70 \\
\hline 16 & 0.08 & 0.14 & 0.10 & 0.53 \\
\hline 17 & 0.08 & 0.07 & 0.12 & 0.92 \\
\hline 18 & $*$ & $*$ & 0.08 & 0.35 \\
\hline
\end{tabular}

$\mathrm{PB}=$ Peripheral Blood

$\mathrm{CB}=$ Central Blood

$\mathrm{ND}=$ Not Detected

* Not analyzed due to interference

Table 3: Postmortem Distribution of Amphetamine (mg/L) or ( $\mathrm{mg} / \mathrm{kg})$.

\begin{tabular}{|c|c|c|c|}
\hline Case \# & $\mathrm{CB} / \mathrm{PB}$ & Vitreous/PB & Liver/PB \\
\hline 1 & 2.00 & 1.40 & 7.80 \\
\hline 2 & - & - & - \\
\hline 3 & 1.25 & 2.25 & - \\
\hline 4 & 1.20 & 1.20 & 3.40 \\
\hline 5 & 1.00 & 2.00 & 7.00 \\
\hline 6 & 1.50 & 2.00 & 7.00 \\
\hline 7 & 0.50 & 2.25 & 17.00 \\
\hline 8 & 2.00 & 3.67 & 14.67 \\
\hline 9 & 2.50 & 3.25 & 11.75 \\
\hline 10 & 2.00 & 2.50 & 8.50 \\
\hline 11 & 1.56 & 0.67 & 2.78 \\
\hline 12 & 2.50 & 1.00 & 5.25 \\
\hline 13 & 1.83 & 1.50 & 6.67 \\
\hline 14 & 1.43 & 1.57 & 7.00 \\
\hline 15 & 1.44 & 1.06 & 4.38 \\
\hline 16 & 1.75 & 1.25 & 6.63 \\
\hline 17 & 0.88 & 1.50 & 11.50 \\
\hline 18 & - & - & - \\
\hline mean & 1.58 & 1.82 & 8.09 \\
\hline SD & 0.55 & 0.82 & 4.03 \\
\hline median & 1.53 & 1.54 & 7.00 \\
\hline
\end{tabular}

$\mathrm{PB}=$ Peripheral Blood

$\mathrm{CB}=$ Central Blood

- no value; not included in statistical analysis

Table 4: Amphetamine Distribution Ratios.

were generally less than twice the level of peripheral blood samples. There were only three cases in which the vitreous methamphetamine levels were lower than peripheral blood. In only one case was amphetamine vitreous level lower than the corresponding peripheral blood concentration.

Methamphetamine liver levels were approximately 6 times that of peripheral blood. The liver to peripheral blood ratio for methamphetamine ranged between 1.94 and 9.14 (mean $5.68 \pm 2.32$ ).

Liver amphetamine averaged 8.1 times peripheral blood levels and showed greater variance than liver methamphetamine levels. The liver to peripheral blood ratio for amphetamine ranged from 2.78 to 17.0 (mean $8.1 \pm 4.0$ ).

These data suggest that in cases where blood is not available, it is helpful to have a liver or vitreous concentration. These specimens may be used to approximate blood levels and assist with interpretation in the absence or contamination of a blood specimen. While $\mathrm{CB} / \mathrm{PB}$ ratios are usually explained by postmortem redistribution, the vitreous/PB ratios may be attributed to a greater affinity of the drug to vitreous.

\section{References}

1. Physician's Desk Reference 2006. PDR. Mason, $\mathrm{OH}$.

2. Baselt RC (2008) Disposition of Toxic Drugs and Chemicals in Man Eighth Edition, Biomedical Publications

3. Barnhart FE, Fogacci JR, Reed DW (1999) Methamphetamine-a study of postmortem redistribution. J Anal Toxicol 23: 69-70.

4. Foerster EH, Hatchett D, Garriott JC (1978) A Rapid, Comprehensive Screening Procedure for Basic Drugs in Blood or Tissues by Gas Chromatography. J Anal Toxicology 2: 50-55. 